\title{
Radical cystectomy for clinical T4b urothelial carcinoma: An Ontario, single-center experience
}

\author{
Daniel Tesolin¹; Asmaa Ismail²; Hazem Elmansy²; Walid Shahrour²; Owen Prowse²; \\ Ahmed Kotb ${ }^{2}$ \\ ${ }^{1}$ Northern Ontario School of Medicine, Thunder Bay, ON, Canada; ${ }^{2}$ Urology Department, Thunder Bay \\ Regional Health Sciences Centre, Thunder Bay, ON, Canada
}

Cite as: Tesolin D, Ismail A, Elmansy H, et al. Radical cystectomy for clinical T4b urothelial carcinoma: An Ontario single-center experience. Can Urol Assoc J 2021 December 21; Epub ahead of print. http://dx.doi.org/10.5489/cuaj. 7277

Published online December 21, 2021

Corresponding author: Dr. Ahmed Kotb, Urology Department, Thunder Bay Regional Health Sciences Centre, Thunder Bay, ON, Canada; drahmedfali@gmail.com

$* * *$

\section{Abstract}

Introduction: Guidelines surrounding the management of $\mathrm{T} 4 \mathrm{~b}$ muscle-invasive bladder cancer (MIBC) with radical cystectomy (RC) are limited and lack clarity. Our objective was to analyze our single-center experience to provide additional insight into the role of RC. Methods: We performed a retrospective data analysis using clinical, radiological, and pathological information for all patients managed by RC for cT4b MIBC at the Thunder Bay Regional Health Sciences Centre (July 2015 to July 2020). Patients that had MIBC as their first diagnosis were termed the de novo group and patients that were initially diagnosed as having non-MIBC were termed the progressive group.

Results: Nineteen consecutive patients (16 males and three females), with a median age of 68 , managed by two urologists over the last five years, met study criteria. Eleven (58\%) of the patients had de novo MIBC while eight (42\%) presented with progressive disease. All patients had dysuria as a presenting symptom. Only one (5\%) patient received neoadjuvant chemotherapy. There were low rates of perioperative transfusion (11\%), bowel resections $(5 \%)$, postoperative transfusions $(0 \%)$, ileus $(32 \%)$, urine leak $(16 \%)$, and wound dehiscence (5\%). Fourteen (74\%) had positive lymph nodes. All patients had adjuvant chemotherapy. The one-year recurrence rate in these patients was $53 \%$, with $32 \%$ of recurrence being distant metastasis. The one-year survival rate was $95 \%$.

Conclusions: Patients in the de novo and progressive arms of our cohort had similar rates of surgical complications and disease recurrence. We found operative morbidity and disease control to be reasonable, suggesting $\mathrm{RC}$ can be considered more often in the management of T4b MIBC patients. 


\section{Introduction}

Radical cystectomy (RC) is the primary treatment option for patients with muscle invasive bladder cancer (MIBC) as supported by the Canadian Urological Association and National Comprehensive Cancer Network (NCCN) guidelines. ${ }^{1,2}$ However, guidelines surrounding the management of T4b MIBC are limited and lack clarity. Due to the uncommon incidence of T4b MIBC, and the high degree of mortality that is usually associated, these patients are excluded from robust treatment trials. ${ }^{3,4} \mathrm{RC}$ in this population can be associated with significant morbidity, while the benefit for cancer control is uncertain.

Urologists would agree that the management of T4b bladder cancer patients should be multidisciplinary, however, there are no clear guidelines on their proper management. The European Association of Urology and American Society of Clinical Oncology endorsed the beneficial role of neoadjuvant chemotherapy (NAC) in patients with cT2- T4a MIBC, without referring to patients with T4b disease. ${ }^{5}$ The Canadian Urological Association guidelines, however, do recommend NAC for unresectable T4b bladder cancer. ${ }^{1} \mathrm{As}$ mentioned above, the precise benefit of NAC in this population is unknown due to the omission of $\mathrm{T} 4 \mathrm{~b}$ patients from clinical trials.

Few reports assess the outcomes of $\mathrm{RC}$ in $\mathrm{T} 4 \mathrm{~b}$ cohorts. Herr et al report that longterm survival is achievable in 32 select patients who received NAC prior to RC. ${ }^{6}$ Black et al reported their experience in managing $23 \mathrm{~T} 4 \mathrm{~b}$ patients and demonstrated acceptable surgical outcomes and good survival. ${ }^{7}$ Approximately $83 \%$ of these patients also received NAC. Nagele et al reported on a German cohort of seven patients with T4b bladder cancer, who did not receive NAC, and concluded primary RC without NAC was technically feasible, with acceptable overall survival. ${ }^{8}$

The literature supporting RC for T4b MIBC is sparse and guidelines are both unclear and inconsistent with their recommendations. Further evidence supporting the role of $\mathrm{RC}$ in the treatment of $\mathrm{T} 4 \mathrm{~b} \mathrm{MIBC}$ is required. The aim of our study was to report our single centre experience in the management of patients with $\mathrm{cT} 4 \mathrm{~b}$ bladder cancer and provide additional insight into the role that RC plays.

\section{Methods}

We performed a retrospective data analysis for all patients managed by RC for cT4b MIBC over the last 5 years. We included patients that had at least 1 year of follow up after surgery as well as patients that died within the first postoperative year. All patients were from the region of Northern Ontario and were treated at the Thunder Bay Regional Health Sciences Centre in Thunder Bay, Ontario, Canada (July 2015- July 2020). Ethical approval was obtained.

We collected clinical, radiological and pathological information for all studied patients. Patients that had MIBC as their first diagnosis were termed the de novo group and patients that were initially diagnosed as having non muscle invasive bladder cancer (NMIBC) were termed the progressive group.

All patients that were diagnosed as having cT4b bladder cancer were discussed in our tumour board meetings that normally convenes on a weekly basis. At that time, all patients' 
radiological scans were reviewed by our radiologists and other members of the board to provide a clinical stage. NAC was not started for patients deemed to be fit and who would benefit more from primary RC in terms of local symptom control.

Two urologists in the hospital were responsible for managing these patients and both urologists did all the cases together. Midline incision below the level of umbilicus was always done. Dissection was usually done in an antegrade fashion from the point of pelvic wall invasion. At this point, complete vascular skeletonization was done, followed by urethral dissection and retrograde mobilization of the bladder. Then, the bladder was dissected with a piece of the attached pelvic wall. If an indurated area was identified at the pelvic wall, at the end of cystectomy, we resected it and submitted the specimen separately for pathological analysis. In all patients, lymph nodes dissection was carried out to the level of the common iliac artery bifurcation, and only extended to aortic bifurcation if there was suspected lymph nodes invasion around common iliac vessels, based on the operators' judgement.

\section{Results}

This study included 19 consecutive patients (16 males and 3 females) managed by two urologists over the last 5 years (July 2015- July 2020). The median age of the patients was 68 years (55- 81 years). Eight patients (42\%) were older than 70 years at time of surgery, including 2 patients older than 80 years old (Table 1 ).

$\mathrm{RC}$ was done for radiologically suspected $\mathrm{T} 4 \mathrm{~b}$ cancer invading the pelvic wall. Eleven of these patients had de novo MIBC while 8 had progressive MIBC after being followed for a median of 2 years (6- 36 months) for NMIBC. All patients in the progressive group had at least one full-dose induction course of BCG. Four patients $(21 \%)$ had unilateral or bilateral nephrostomy tube insertions before surgery because of urinary tract obstruction and worsening renal function.

The most common presenting symptom for these patients was dysuria rather than hematuria. All de novo group patients had dysuria as the sole presenting symptom and were treated empirically for urinary tract infection with antibiotics before being referred to urology. For the progressive group, 8 patients had significant dysuria, with 4 of them reporting intermittent episodes of gross hematuria as well. Figures 1- 3 represent selected pelvic CT images of the bladder cancer patients preoperatively.

NAC was only given to a single patient in the de novo group, as other patients had significant local pain and RC was advocated as a primary treatment, omitting NAC, mainly for local symptoms control. All patients had adjuvant chemotherapy (Cisplatin and Gemcitabine). The median time from cystectomy to adjuvant chemotherapy was 8 weeks (218 weeks).

On preoperative scans, no patients in the progressive group had suspected pelvic lymphadenopathy. In the de novo group, lymphadenopathy was suspected within the obturator and internal iliac regions in three patients $(15.7 \%)$.

The median surgical time was 4 hours (3-5). Ileal conduit urine diversion was used for all patients. Two patients (10.5\%) required intraoperative blood transfusions of 2 units. Both patients were females. No patients required postoperative blood transfusions. One 
patient $(5 \%)$ had their tumour infiltrating the rectum, requiring partial resection of the anterior rectal wall and a temporary colostomy. The median hospital stay was 14 days (1021). Early postoperative complications were ileus (6 patients $31.5 \%$ ), urine leak ( 3 patients $15.7 \%$ ) and wound dehiscence (1 patient 5\%).

Postoperative lymph node (LN) pathological analysis revealed 14 patients (74\%) had positive pelvic LN invasion, including 8 from the de novo group and 6 from the progressive group. The LN regions involved were the obturator, internal iliac and external iliac chains. Common iliac LNs were only positive in 2 patients. Out of the 16 men, 6 patients $(37.5 \%)$ had associated prostate cancer; including 2 patients with Gleason 3+3, 2 patients with $3+4$ and 2 patients with $4+3$ disease.

Positive surgical margins were identified in 2 patients from the progressive group and 4 patients in the de novo group.

The median postoperative follow up was 18 months (3-30). Recurrence occurred in 10 patients $(53 \%)$ by the end of the first postoperative year. This included 4 patients that had local recurrence which presented as an enhanced mass in the pelvic wall and 6 patients that had distant metastases to lungs and/or liver. Recurrence occurred for 5 patients from each group. All recurred patients had positive LN invasion and 3 of them had positive surgical margin at time of cystectomy. Recurrence free survival was $47 \%$ at the end of the first and second-year post surgery. No additional patients developed recurrence during the second year of follow up. By the end of the first year, one patient died (5\%). Three other patients $(15.7 \%)$ died during further follow up beyond 1 year. The overall survival was $95 \%, 84 \%$ and $79 \%$ at 1,2 and 3 years respectively.

\section{Discussion}

Our results demonstrate that $\mathrm{RC}$ is a reasonable treatment option for patients with $\mathrm{T} 4 \mathrm{~b} \mathrm{MIBC}$. Although surgical extirpation is challenging, acceptable rates of complications were observed, with most complications being minor. Despite the diagnosis of locally advanced cancer, nearly half of the patients demonstrated no evidence of recurrence after 1-year follow-up post RC. The complication rate and disease control were similar amongst the progressive and de novo groups. All patients but one did not receive NAC. The question about the absolute benefits for routine NAC in these patients are not answered due to the structure of this study.

Painless hematuria is usually the most common presenting symptom reported for bladder cancer ${ }^{9}$. This does not seem to be the case for our cohort where none of the patients in the de novo group had hematuria. Only half the patients presented with hematuria in the progressive group. The most predominant presenting symptom exhibited by our patients was dysuria. In fact, most patients were treated empirically for urinary tract infections before being referred to a urologist. This highlights a different pattern of presentation in patients with advanced bladder cancer versus early disease.

The rates of peri-operative and post-operative complications in our patients were modest when considering the degree of difficulty of the procedure. The rates of complications were similar between the progressive and the de novo group. 
Pathological LN involvement was frequent, but similar amongst both the progressive and de novo groups ( $75 \%$ vs $73 \%$ ). This is not surprising given the advanced nature of disease in these patients. The rate of overall recurrence within 1 year was reasonable at $53 \%$. The rate of recurrence was also similar between both groups. The proportion of patients with distant metastasis within 1 year post-operatively was $32 \%$. This suggests that RC was efficacious in controlling the short-term course of disease in nearly half of these patients that would have otherwise been treated with palliative intent. This correlates with a retrospective analysis of the SWOG 8710 Trial reporting a 5-year survival for T3-4 of $22 \%{ }^{10}$ These results also suggest that patients who present with progressive versus de novo disease should not be treated differently.

Some consensus guidelines endorse the utilisation of NAC for T4b patients. Only a single patient in the de novo group was treated with NAC. All patients received adjuvant chemotherapy. The median time from RC to adjuvant chemotherapy was 8 weeks. Our results demonstrate acceptable disease control despite most patients not receiving NAC.

This study was designed to add clarity to treatment guidelines by examining the role of RC in the treatment of T4b MIBC. Often studies suggest patients should be carefully selected for RC given the associated morbidity, however, $42 \%$ of the patients in our cohort were over 70 years-old, suggesting RC can be an effective option, even for elderly patients. Also, in our cohort population, only one patient received NAC. Despite some guidelines suggesting RC should be reserved for patients who respond to NAC, Nagele et al postulate that if patients do not respond to NAC, their benefit from RC is reduced due to the risk of disease progression from treatment delay. ${ }^{8}$ A retrospective analysis of 896 patients with $\mathrm{T} 4 \mathrm{~b}$ N0-3 M0 from the National Cancer Database suggests that the receipt of RC was associated with overall survival 14.2 months, regardless of other treatments that patients received. ${ }^{11}$ This is higher than overall survival associated with treatment using chemotherapy or chemoradiation. Perhaps RC should be considered more readily for a more general population of patients with T4b MIBC.

It should be known that this report is not novel and that our findings are consistent with similar studies. However, there are no studies that are representative of the Northwestern Ontarian region, a population which tends to be more medically complex compared to those of larger, urban centres. ${ }^{12}$ Although the power of this study is limited by sample size, the incidence of T4b MIBC is low and therefore the sample size in this study is reasonable compared to other studies examining similar populations. This study is also limited by being retrospective in nature, possibly allowing the introduction of unmeasured confounding factors.

\section{Conclusions}

Certainly, more studies are required to elucidate the exact role of RC in T4b MIBC. Our results suggest primary $\mathrm{RC}$ is feasible with limited morbidity and excellent clinical response, even in elderly patients. This remains true for patients who present with progressive and $d e$ novo disease. Interestingly, all patients presented with dysuria, but only half with hematuria. Both groups had experienced similar morbidity and disease control from RC suggesting these 
groups should be treated the same. We believe our findings demonstrate a need for robust trial surrounding the role of $\mathrm{RC}$ in $\mathrm{T} 4 \mathrm{~b} \mathrm{MIBC}$ as its role may be underemphasized in the guidelines.

\section{References}

1. Kulkarni GS, Black PC, Sridhar SS, et al. Canadian Urological Association guideline: Muscle-invasive bladder cancer. Can Urol Assoc J. 2019;13(8). doi:10.5489/cuaj.5902

2. NCCN Clinical Practice Guidelines in Oncology (NCCN Guidelines(R)). Bladder Cancer. Published online Version 2020. Accessed September 2, 2021. https://www.nccn.org/professionals/physician_gls/pdf/bladder.pdf

3. Grossman HB, Natale RB, Tangen CM, et al. Neoadjuvant Chemotherapy plus Cystectomy Compared with Cystectomy Alone for Locally Advanced Bladder Cancer. N Engl J Med. 2003;349(9):859-866. doi:10.1056/NEJMoa022148

4. Sherif A, Holmberg L, Rintala E, et al. Neoadjuvant Cisplatinum Based Combination Chemotherapy in Patients with Invasive Bladder Cancer: A Combined Analysis of Two Nordic Studies. Eur Urol. 2004;45(3):297-303. doi:10.1016/j.eururo.2003.09.019

5. Milowsky MI, Rumble RB, Booth CM, et al. Guideline on Muscle-Invasive and Metastatic Bladder Cancer (European Association of Urology Guideline): American Society of Clinical Oncology Clinical Practice Guideline Endorsement. J Clin Oncol. 2016;34(16):1945-1952. doi:10.1200/JCO.2015.65.9797

6. Herr HW, Donat SM, Bajorin DF. Post-chemotherapy surgery in patients with unresectable or regionally metastatic bladder cancer. J Urol. 2001;165(3):811-814.

7. Black PC, Dinney CPN, Brown GA, et al. The role of radical cystectomy in patients with clinical T4b bladder cancer. Urol Oncol Semin Orig Investig. 2011;29(2):157161. doi:10.1016/j.urolonc.2009.12.015

8. Kuczyk M, Nagele U, Anastasiadis A, et al. Surgical approach in patients with T4 bladder cancer as primary treatment: Disaster or option with improved quality of life. Indian J Urol. 2008;24(1):95. doi:10.4103/0970-1591.38610

9. Pashos CL, Botteman MF, Laskin BL, Redaelli A. Bladder Cancer. Cancer Pract. 2002;10(6):311-322. doi:10.1046/j.1523-5394.2002.106011.x

10. Herr HW, Faulkner JR, Grossman HB, et al. Surgical Factors Influence Bladder Cancer Outcomes: A Cooperative Group Report. J Clin Oncol. 2004;22(14):27812789. doi:10.1200/JCO.2004.11.024

11. Haque W, Verma V, Butler EB, Teh BS. National Practice Patterns and Outcomes for T4b Urothelial Cancer of the Bladder. Clin Genitourin Cancer. 2018;16(1):4249.e1. doi:10.1016/j.clgc.2017.08.013

12. Romanow RJ. Buildings on Values: The Future of Health Care in Canada : Final Report. Commission on the Future of Health Care in Canada; 2002. 


\section{Figures and Tables}

Fig. 1. A 63-year-old man with a large bladder cancer infiltrating the left pelvic side wall.

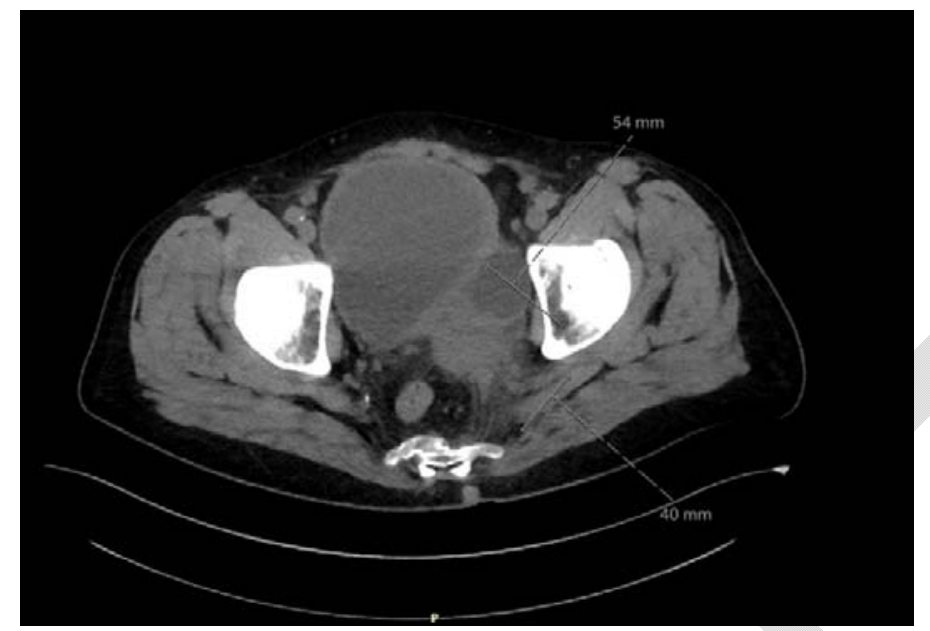

Fig. 2. A 74-year-old man with a large tumour infiltrating both pelvic side walls, more on left side.

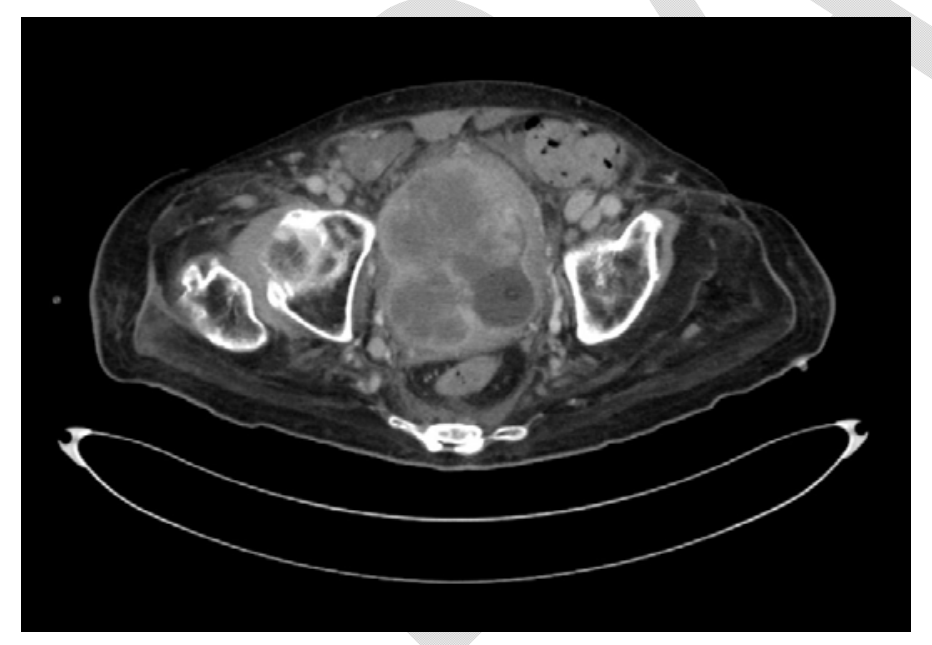


Fig. 3. A 78-year-old lady with a large tumour infiltrating left pelvic side wall.

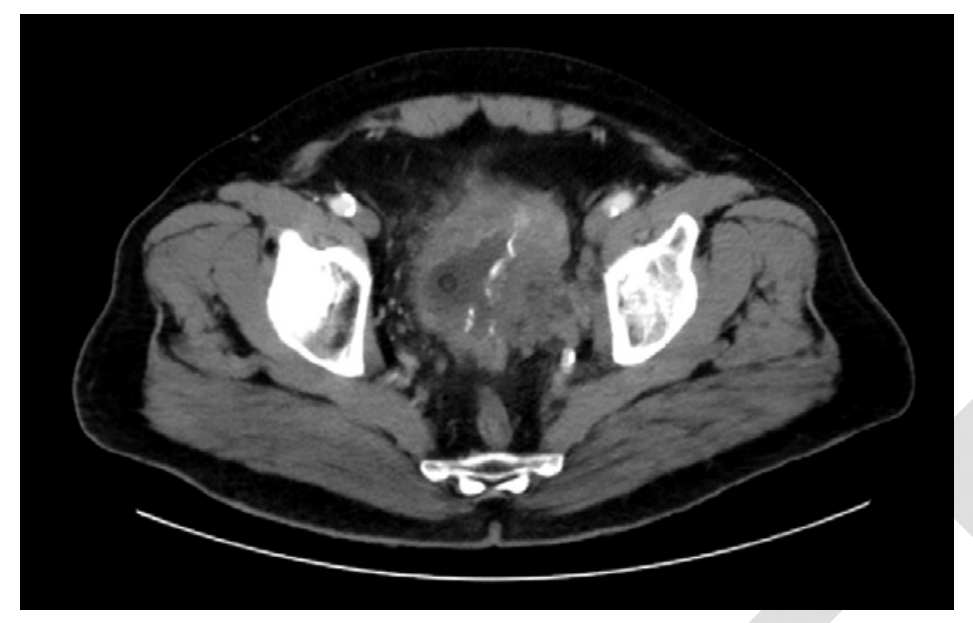

\begin{tabular}{|l|l|}
\hline \multicolumn{2}{|l|}{ Table 1. Clinicopathological and demographic patient characteristics } \\
\hline Characteristic, n (\%) & Total \\
\hline Demographics & $68(55-81)$ \\
Median age (years) & $16(84)$ \\
Male & $3(16)$ \\
Female & $19(100)$ \\
Total & \\
\hline Preoperative issues & $19(100)$ \\
Dysuria & - \\
Hematuria & $4(21)$ \\
Nephrostomy tube placement & \\
\hline Perioperative complications & $4(3-5)$ \\
Median surgical time (hours) & $2(11)$ \\
Transfusion & $1(5)$ \\
Bowel resection/colostomy & \\
\hline Postoperative complications & $0(0)$ \\
Transfusion & $6(32)$ \\
Ileus & $3(16)$ \\
Urine leak & $1(5)$ \\
Wound dehiscence & 14 \\
Median hospital stay (days) & \\
\hline Lymph node involvement & $14(74)$ \\
Positive node(s) & $6(32)$ \\
\hline Prostate cancer-associated & \\
Positive & $18(3-30)$ \\
\hline Followup & $10(53)$ \\
Median followup (months) & $6(32)$ \\
1-year any recurrence & $18(95)$ \\
1-year distant metastasis & $19(100)$ \\
1-year survival & \\
Adjuvant chemotherapy & \\
\hline
\end{tabular}




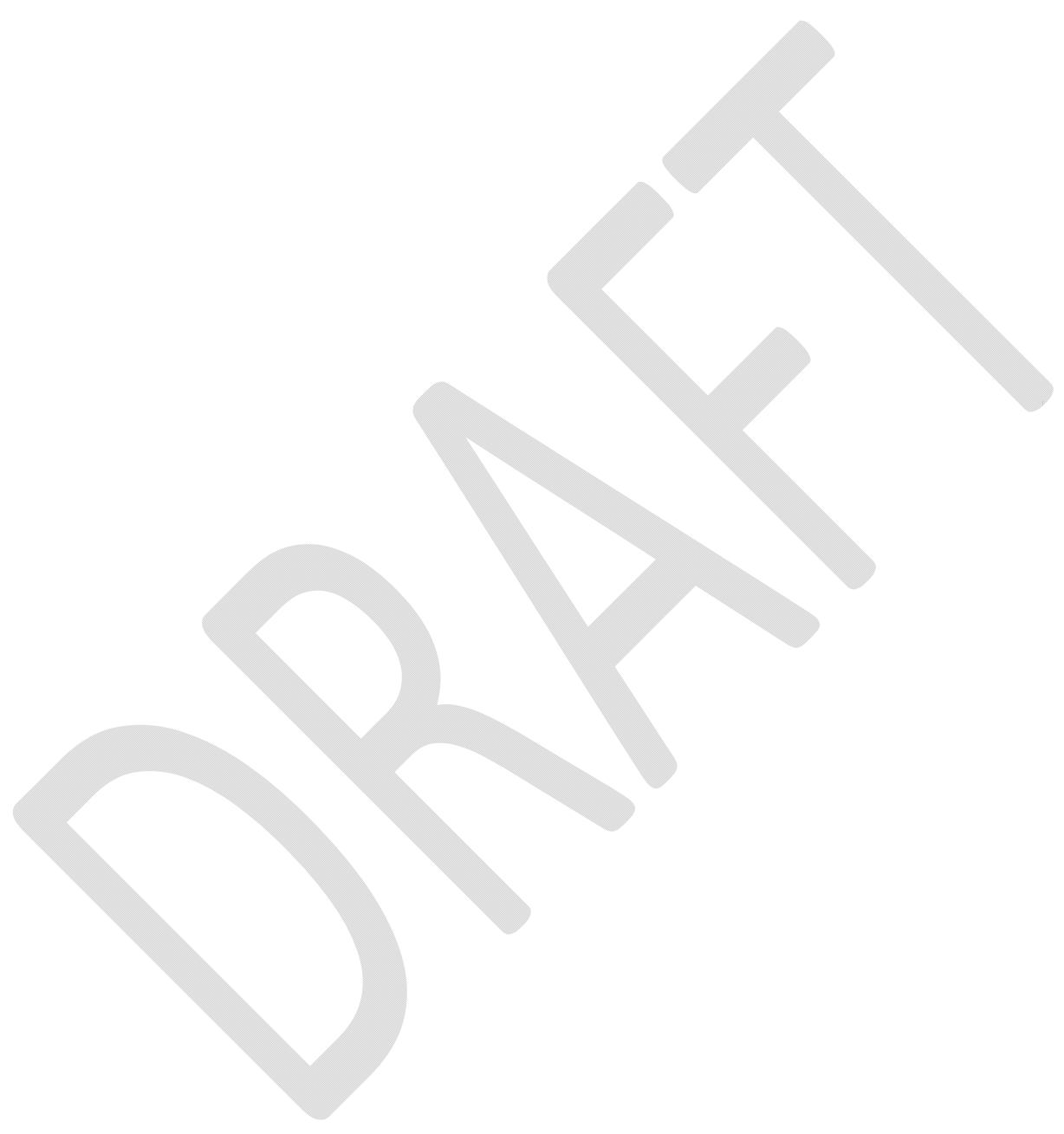

